\title{
Pre-pregnancy potato consumption and risk of gestational diabetes mellitus: prospective cohort study
}

\author{
Wei Bao, ${ }^{1}$ Deirdre K Tobias, 2,3 Frank B Hu, ${ }^{3,4,5}$ Jorge E Chavarro, ${ }^{3,4,5}$ Cuilin Zhang' ${ }^{1}$
}

'Epidemiology Branch, Division of Intramural Population Health Research, Eunice Kennedy Shriver National Institute of Child Health and Human

Development, National Institutes of Health, Rockville, MD 20852, USA

2Division of Preventive Medicine, Brigham and Women's Hospital and Harvard Medical School, Boston, MA 02215, USA

${ }^{3}$ Department of Nutrition, Harvard TH Chan School of Public Health, Boston, MA

02115, USA

4Department of Epidemiology, Harvard TH Chan School of Public Health, Boston, MA

02115, USA

${ }^{5}$ Channing Division of Network Medicine, Department of Medicine, Brigham and Women's Hospital and Harvard Medical School, Boston, MA 02115, USA

Correspondence to: C Zhang zhangcu@mail.nih.gov

Additional material is published online only. To view please visit the journal online (http://dx.doi. org/10.1136/bmj.h6898)

Cite this as: BMJ 2016;352:h6898 http://dx.doi.org/10.1136/bmj.h6898

Accepted: 03 December 2015

\section{ABSTRACT}

\section{STUDY QUESTION}

What is the association between potato consumption before pregnancy and the risk of gestational diabetes mellitus (GDM)?

\section{METHODS}

This prospective cohort study included 15632 women from the Nurses' Health Study II (1991-2001). They had no previous GDM or chronic diseases before pregnancy. Consumption of potatoes and other foods was assessed every four years. Incident first time GDM was ascertained from self reports of a physician diagnosis of GDM, which was previously validated by medical records.

\section{STUDY ANSWER AND LIMITATIONS}

Over the 10 year follow-up there were 854 incident cases of GDM among 21693 singleton pregnancies. After adjustment for age, parity, and dietary and non-dietary factors, women who consumed more potatoes before pregnancy had higher rates of developing GDM. Substitution of two servings a week of total potatoes with other vegetables, legumes, and whole grain foods was significantly associated with a 9-12\% lower risk of GDM. Consumption and diabetes were self reported, and severity of diabetes was unknown. More than $90 \%$ of women were white. A causal association cannot be assumed.

\section{WHAT THIS PAPER ADDS}

Higher levels of potato consumption before pregnancy are associated with greater risk of GDM, and substitution of potatoes with other vegetables, legumes, or whole grain foods might lower the risk. FUNDING, COMPETING INTERESTS, DATA SHARING Funding was received from the Intramural Research Program of the Eunice Kennedy Shriver National Institute of Child Health and Human Development,

\section{WHAT IS ALREADY KNOWN ON THIS TOPIC}

Gestational diabetes is a common complication of pregnancy and is at the center of a vicious circle of diabetes-begets-diabetes across generations

The Dietary Guidelines for Americans continuously include potatoes within the vegetable food group as a healthy food, though previous studies suggest that potatoes can adversely affect glucose metabolism because they contain large amounts of rapidly absorbable starch

The association between potato consumption and risk of gestational diabetes mellitus remains unknown

\section{WHAT THIS STUDY ADDS}

Pre-pregnancy potato consumption was significantly and positively associated with the risk of GDM

Substitution of potatoes with other vegetables, legumes, or whole grain foods might lower the risk of GDM the National Institutes of Health, and the American Diabetes Association (No 7-12-MN-34). The authors have no competing interests or additional data to share.

\section{Introduction}

Potatoes are the third most commonly consumed food crop in the world, after rice and wheat. ${ }^{1}$ In the United States, about 35\% of women of reproductive age (that is, aged 19-50) consume potatoes daily, accounting for $8 \%$ of daily total energy intake. ${ }^{2}$ The health effects of potatoes are inconclusive, ${ }^{13}$ and there have been longstanding debates on the appropriate placement of potatoes in dietary guidance. ${ }^{4-6}$ Nonetheless, the Dietary Guidelines for Americans continue to include potatoes in the vegetable food group and encourage consumption. ${ }^{7}$ Though potatoes are rich in vitamin $\mathrm{C}$, potassium, dietary fiber, and some phytochemicals, ${ }^{6}$ unlike other vegetables they can have detrimental effects on glucose metabolism because they contain large amounts of rapidly absorbable starch. ${ }^{3}$ Indeed, several epidemiologic studies have linked higher potato consumption to increased concentrations of fasting plasma glucose, ${ }^{8}$ insulin resistance, ${ }^{8}$ and an increased risk of type 2 diabetes mellitus. ${ }^{910}$

Gestational diabetes mellitus (GDM) is a common complication of pregnancy characterized by glucose intolerance with onset or first recognition during pregnancy. ${ }^{11}$ GDM is not only associated with adverse perinatal outcomes, ${ }^{12}$ it is also related to increased long term cardiometabolic risk in both mothers and their offspring. ${ }^{113}$ It is therefore crucial to identify modifiable risk factors that could contribute to the prevention of GDM. Previous studies have found that a diet with a higher glycemic index is related to higher plasma glucose and HbA1c concentrations during pregnancy ${ }^{1415}$ and could increase the risk of GDM. ${ }^{16}$ The association between consumption of potatoes, a commonly consumed food with a high glycemic index, and GDM risk, remains unknown. Using data from a large prospective cohort study, we examined the association between potato consumption before pregnancy and risk of incident GDM.

\section{Methods}

\section{Study population}

The Nurses' Health Study II (NHSII) is an ongoing prospective cohort study of 116430 female nurses aged 24-44 at study inception in 1989.17 The participants received a biennial questionnaire regarding disease outcomes and lifestyle behaviors, such as smoking status and medication use. Follow-up for each questionnaire cycle was greater than $90 \%{ }^{18}$ 
We included participants from NHSII in this analysis if they reported at least one incident singleton pregnancy that lasted more than six months. The 1991 questionnaire was the first time dietary information was captured. We therefore set this year as the baseline for this analysis and included only pregnancies occurring after the return of 1991 questionnaire. The 2001 questionnaire was the last time GDM was ascertained as most participants had passed reproductive age by then; thus, length follow-up of GDM was up to the return of the 2001 questionnaire. Individual pregnancies were eligible if the woman did not report GDM in a previous pregnancy and had no prior diagnosis of type 2 diabetes, cardiovascular disease, or cancer. We excluded pregnancies if the woman did not return at least one pre-pregnancy food frequency questionnaire, left more than 70 questionnaire items blank, or reported unrealistic total energy intake $(<2510$ or $>14644 \mathrm{~kJ} /$ day, equivalent to $<600$ or $>3500 \mathrm{kcal} /$ day). We excluded women with GDM in a previous pregnancy because they might change their diet and lifestyle during the next pregnancy to prevent recurrence.

\section{Assessment of exposure}

Beginning in 1991 and every four years thereafter, study participants reported their usual food intake during the previous year on a food frequency questionnaire. The validity and reproducibility of the questionnaire are documented elsewhere. ${ }^{19-21}$ For potato consumption, we asked each participant how often, on average, they had consumed baked, boiled, or mashed potatoes (serving size: one baked or boiled or $237 \mathrm{~mL}$ (equivalent to one cup) mashed) or French fries (serving size $113 \mathrm{~g}$ ) during the previous year. The possible responses of frequency ranged from never or less than once/month to six or more times a day. In a previous validation study, the corrected correlations between the food frequency questionnaire and the diet records were 0.66 for potatoes and 0.60 for French fries. ${ }^{21}$ We summed consumptions of baked, boiled, or mashed potatoes and French fries as total potato consumption. In addition, we asked the participants their frequency of consumption of potato chips or corn chips (combined) during the previous year (serving size 28 g or small bag).

\section{Ascertainment of outcomes}

The NHSII participants reported incident GDM on each biennial questionnaire through 2001. For women who reported more than one pregnancy lasting more than six months within a two year questionnaire period, GDM status was attributed to the first pregnancy. In a prior validation study among a subgroup of the NHSII cohort, 94\% of GDM self reports were confirmed by medical records. ${ }^{17}$ In a random sample of parous women without GDM, 83\% reported undergoing a glucose screening test during pregnancy and 100\% reported frequent prenatal urine screenings, which indicated a high level of GDM surveillance in this cohort. ${ }^{17}$ During the period that GDM events were reported in the present study (1991-2001), the National
Diabetes Data Group criteria ${ }^{22}$ have been widely adopted for GDM diagnosis.

\section{Assessment of covariates}

Participants reported their height and weight in 1989 and updated their weight on each biennial questionnaire. In a previous validation study, self reported weight was highly correlated with weight measured by a technician ( $r=0.97) .{ }^{23}$ Body mass index (BMI) was computed as weight in kilograms divided by height in meters squared. Total physical activity was ascertained by frequency of engaging in common recreational activities, from which metabolic equivalent hours per week were derived. A prior validation study showed that questionnaire based estimates correlated well with detailed activity diaries $(r=0.56){ }^{24}$

To account for potential confounding by the participants' overall quality of diet, we derived a diet score-the alternate healthy eating index 2010 (AHEI-2010)-for each participant, as previously described. ${ }^{25}$ In brief, we allotted 0 to 10 points for intake of each of the following components, with adherence to dietary recommendations receiving 10 points: vegetables (servings/day), fruits (servings/day), whole grains (g/day), sugar sweetened beverages and fruit juice (inversely scored, servings/day), nuts and legumes (servings/day), red/ processed meat (inversely scored, servings/day), trans fat (inversely scored, \% of energy), long chain n-3 fats (mg/day), polyunsaturated fat (\% of energy), sodium (inversely scored, mg/day), and alcohol (drinks/day; we assigned the highest score to moderate (0.5-1.5 drinks/ day) and the worst score to heavy ( $\geq 2.5$ drinks/day) alcohol consumers). The overall AHEI-2010 ranged from 0 to 110 points, with a higher score indicating a better quality of diet and associated with a lower risk of incident chronic diseases, including cardiovascular disease, diabetes, and cancer. ${ }^{25}$

\section{Statistical analysis}

We calculated a cumulative average intake of potato consumption before pregnancy for each individual at each time period to reduce variation within individuals and to represent long term habitual pre-pregnancy diet. ${ }^{26}$ For instance, the 1991 intake was used for the follow-up between 1991 and 1995, the average of the 1991 and 1995 intake was used for the follow-up between 1995 and 1999, and so on.

Descriptive statistics at baseline are presented as means (standard deviations) for continuous variables and percentages for categorical variables. To compare categories of pre-pregnancy potato consumption, we used analysis of variance test for continuous variables and $\chi^{2}$ test for categorical variables. We used log-binomials models ${ }^{27}$ with generalized estimating equations to estimate relative risks and $95 \%$ confidence intervals. Generalized estimating equations allowed us to account for correlations among repeated observations (pregnancies) contributed by a single participant. ${ }^{28}$ In a few instances, the log-binomials models did not converge, and we used log-Poisson models, ${ }^{29}$ which provide consistent but not fully efficient risk estimates. 
Pre-pregnancy covariates in the multivariable models included age (continuous), parity $(0,1,2$, and $\geq 3$ pregnancies lasting six or more months), race/ethnicity (white, African-American, Hispanic, Asian, other, and missing/not reported), family history of diabetes (yes, no), cigarette smoking (never, past, current, and missing/not reported), physical activity (fourths), total energy intake (fourths), and overall diet quality as represented by the AHEI-2010 dietary pattern score (fourths). We additionally adjusted for biennially updated measures of BMI (<21, 21-22.9, 23.0-24.9, 25.026.9, 27.0-28.9, 29.0-30.9, 31.0-32.9, 33.0-34.9, 235.0). BMI was entered into the model separately because it can also be an intermediate on the pathway between potato consumption and GDM risk. We derived covariates from the questionnaire preceding each pregnancy, except race/ethnicity and family history of diabetes, which were reported in 1989. Categorical covariates included an indicator variable for missing data, if necessary. To tests for linear trends across categories of potato consumption we assigned the median intake for each category and fitted this as continuous variable in the models. In a sensitivity analysis for the tests of linear trends, we used the actual continuous amount of total potato consumption in the analysis.

We estimated the effect of substituting two servings/ week of total potatoes with the same serving amount of alternative foods (that is, other vegetables, legumes, or whole grain foods) by including both as continuous variables in the same multivariable model, which also contained non-dietary covariates and dietary covariates including total energy intake. We estimated relative risks and 95\% confidence intervals for the substitution associations by computing the difference in their $\beta$ coefficients and in their own variances and covariance. ${ }^{30}$

To evaluate effect modification, we performed stratified analyses according to age $(<35 v \geq 35)$, parity (nulliparous $v$ parous), family history of diabetes (yes $v$ no), physical activity before pregnancy $(<$ median $v$ $\geq$ median), overall quality of diet (AHEI-2010 dietary pattern score; $<$ median $v \geq$ median), and overweight status before pregnancy (BMI $<25 v \geq 25$ ). We tested interactions between these factors and potato consumption by adding multiplicative interaction terms of the binary effect modifiers and continuous linear trend variables in the multivariable models.

To assess the robustness of our findings, we performed a series of sensitivity analyses by using different modeling approaches. For instance, in a sensitivity analysis, we adjusted for individual food items related to diabetes (such as red meat, fruits, vegetables, whole grains, and sugar sweetened beverages), instead of the AHEI diet quality score that represents a combination of individual food items as a dietary pattern. ${ }^{25}$ In addition, we performed a sensitivity analysis using the most recent data for potato consumption before pregnancy instead of the cumulative average intake as the exposure variable. We also conducted sensitivity analyses by adjusting for major covariates as more refined categories (such as tenths) or as continuous variables. Among the participants included in this analysis, less than 3\% of covariate data were missing for a given variable. Collectively, only $4.5 \%$ of the total participants had at least one missing variable in the covariates. To examine the impact of incomplete data in our analysis, we conducted a sensitivity analysis by omitting those participants with incomplete covariate data.

All statistical analyses were performed with SAS software (version 9.3; SAS Institute). $\mathrm{P}<0.05$ was considered significant.

\section{Patient involvement}

No patients were involved in setting the research question or the outcome measures, nor were they involved in developing plans for recruitment, design and implementation of the study. No patients were asked to advise on interpretation or writing up of results. There are no plans to disseminate the results of the research to study participants or the relevant patient community.

\section{Results}

During the 10 years of follow-up, we documented 854 incident pregnancies affected by GDM among 21693 eligible singleton pregnancies from 15632 women (6061 women with more than one pregnancy). At baseline in 1991, women with higher potato consumption were younger, less likely to be nulliparous, and more likely to be current smokers (table 1). They had a higher BMI, performed less physical activity, consumed greater total energy, and had a lower quality of overall diet as represented by the AHEI-2010 dietary pattern score.

Higher total potato consumption was significantly associated with an increased risk of GDM (table 2). After adjustment for age, parity, dietary and nondietary factors, the relative risks of GDM for total potato consumption of 1, 2-4, and $\geq 5$ servings/week, compared with $<1$ serving/week, were 1.21 (95\% confidence interval 0.98 to 1.50 ), 1.34 (1.10 to 1.64), and 1.62 (1.24 to 2.13), respectively $(\mathrm{P}<0.001$ for trend). The association was modestly attenuated but remained significant after additional adjustment for BMI, with the corresponding relative risks of 1.20 (0.97 to 1.48), 1.27 (1.04 to 1.55), and 1.50 (1.15 to 1.96 ), respectively ( $\mathrm{P}=0.006$ for trend). Additional adjustment for glycemic index did not alter the association between consumption and risk. In addition, associations did not differ by other risk factors for GDM, such as age, parity, family history of diabetes, physical activity, overall diet quality, or overweight status (data not shown). Substitution of two servings per week of total potatoes with other vegetables, legumes, and whole grain foods was significantly associated with a lower risk of GDM: 9\% (0.91, 0.85 to 0.97$)$ lower risk for other vegetables, $10 \%$ (0.90, 0.83 to 0.99$)$ for legumes, and $12 \%(0.88,0.83$ to 0.94$)$ for whole grain foods (fig 1$)$.

We also examined the association between risk of GDM and different types of potato preparations (tables 3 and 4). The multivariable adjusted relative risks for GDM for baked, boiled, or mashed potato consumption of 1, 2-4, and $\geq 5$ servings/week, compared with $<1$ serving/week, were 1.16 (95\% confidence interval 0.97 to 1.39 ), 1.30 (1.08 to 1.57), and 1.57 (1.14 to 2.15), respectively ( $\mathrm{P}=0.001$ for trend). The association 


\begin{tabular}{|c|c|c|c|c|}
\hline \multirow[b]{2}{*}{ Characteristics } & \multicolumn{4}{|c|}{ Consumption (servings/week) } \\
\hline & $<1$ & 1 & $2-4$ & $\geq 5$ \\
\hline No of participants & 3206 & 4068 & 6832 & 1526 \\
\hline Mean (SD) age (years) & $32.3(3.4)$ & $32.1(3.3)$ & $31.9(3.2)$ & $31.6(3.2)$ \\
\hline White & 2891 (90.3) & 3808 (93.6) & $6433(94.2)$ & $1425(93.3)$ \\
\hline Family history of diabetes & $352(10.8)$ & $431(10.6)$ & $764(11.2)$ & $197(13.1)$ \\
\hline Nulliparous & $1394(43.8)$ & $1468(36.2)$ & $2164(31.6)$ & $449(28.9)$ \\
\hline Current smoking & $232(7.2)$ & $345(8.5)$ & $638(9.4)$ & $174(11.4)$ \\
\hline Alcohol (g/d) & $2.9(5.0)$ & $3.2(5.4)$ & $3.0(5.2)$ & $2.7(4.8)$ \\
\hline BMI & $22.9(4.0)$ & $23.2(4.0)$ & $23.7(4.6)$ & $24.2(4.8)$ \\
\hline $\begin{array}{l}\text { Physical activity (metabolic } \\
\text { equivalent-h/week) }\end{array}$ & $25.5(31.4)$ & $24.7(31.0)$ & $22.0(27.1)$ & $22.0(29.6)$ \\
\hline Total calories (kJ/d) & $6453.8(2048.9)$ & $7176.4(2038.9)$ & 8106.9 (2188.2) & $9559.6(2248.5)$ \\
\hline Carbohydrate (\%E) & $51.2(7.7)$ & $50.7(7.1)$ & $50.3(7.0)$ & $50.3(7.3)$ \\
\hline Protein $(\% \mathrm{E})$ & $19.6(3.6)$ & $19.5(3.4)$ & $19.1(3.1)$ & $18.6(3.1)$ \\
\hline Total fat (\%E) & $29.9(5.6)$ & $30.4(5.3)$ & $31.4(5.3)$ & $32.3(5.7)$ \\
\hline Saturated fat (\%E) & $10.9(2.6)$ & $11.0(2.4)$ & $11.3(2.3)$ & $11.5(2.4)$ \\
\hline Monounsaturated fat (\%E) & $11.1(2.3)$ & $11.4(2.2)$ & $11.9(2.3)$ & $12.5(2.5)$ \\
\hline Polyunsaturated fat (\%E) & $5.4(1.5)$ & $5.4(1.3)$ & $5.5(1.2)$ & $5.5(1.2)$ \\
\hline Trans fat (\%E) & $1.4(0.5)$ & $1.5(0.5)$ & $1.7(0.6)$ & $1.9(0.7)$ \\
\hline Cholesterol (mg/dt) & $232.9(68.8)$ & $236.3(64.3)$ & $240.4(61.0)$ & $238.5(66.0)$ \\
\hline Glycemic indext) & $53.1(3.7)$ & $53.4(3.2)$ & $54.4(2.9)$ & $55.6(2.7)$ \\
\hline Glycemic loadt) & $124.4(22.9)$ & $123.0(20.5)$ & $123.0(19.8)$ & $124.1(20.2)$ \\
\hline Total fiber (g/dt) & $18.1(6.0)$ & $18.2(5.6)$ & $17.9(5.1)$ & $18.2(5.2)$ \\
\hline Magnesium (mg/dt) & $335.4(78.9)$ & $328.7(74.3)$ & $311.0(69.3)$ & $292.6(65.9)$ \\
\hline Heme iron (mg/dt) & $1.0(0.4)$ & $1.1(0.4)$ & $1.1(0.4)$ & $1.1(0.4)$ \\
\hline Potassium (mg/dt) & $2880.1(527.7)$ & $2887.1(510.4)$ & $2879.2(491.8)$ & $2900.6(514.3)$ \\
\hline Calcium (mg/dt) & $1161.4(487.3)$ & $1118.3(426.5)$ & $1036.1(390.2)$ & $940.0(351.4)$ \\
\hline Vitamin C (mg/dt) & $290.4(334.6)$ & $252.0(272.9)$ & $232.0(245.1)$ & $204.0(198.5)$ \\
\hline Vitamin E (mg/dt) & $24.4(54.7)$ & $20.8(46.3)$ & $19.5(40.4)$ & $16.2(29.6)$ \\
\hline Red meat (servings/d) & $0.5(0.4)$ & $0.6(0.5)$ & $0.8(0.5)$ & $1.2(0.7)$ \\
\hline Poultry (servings/d) & $0.4(0.3)$ & $0.4(0.3)$ & $0.5(0.3)$ & $0.5(0.3)$ \\
\hline Fish (servings/d) & $0.2(0.2)$ & $0.2(0.2)$ & $0.2(0.2)$ & $0.2(0.2)$ \\
\hline Eggs (servings/d) & $0.1(0.2)$ & $0.2(0.2)$ & $0.2(0.2)$ & $0.2(0.2)$ \\
\hline Low fat dairy (servings/d) & $1.4(1.3)$ & $1.6(1.3)$ & $1.6(1.2)$ & $1.5(1.3)$ \\
\hline High fat dairy (servings/d) & $0.9(0.9)$ & $0.9(0.9)$ & $1.0(0.9)$ & $1.1(0.9)$ \\
\hline Nuts (servings/d) & $0.2(0.3)$ & $0.2(0.4)$ & $0.3(0.3)$ & $0.3(0.4)$ \\
\hline Legumes (servings/d) & $0.3(0.3)$ & $0.3(0.3)$ & $0.4(0.3)$ & $0.5(0.4)$ \\
\hline Vegetables (servings/d) & $2.7(2.2)$ & $3.0(1.9)$ & $3.3(2.0)$ & $3.6(2.3)$ \\
\hline Fruits (servings/d) & $1.1(0.9)$ & $1.2(1.0)$ & $1.2(1.0)$ & $1.2(1.1)$ \\
\hline $\begin{array}{l}\text { Whole grain foods } \\
\text { (servings/d) }\end{array}$ & $1.3(1.2)$ & $1.4(1.2)$ & $1.5(1.2)$ & $1.6(1.3)$ \\
\hline SSBs (servings/d) & $0.4(0.7)$ & $0.4(0.7)$ & $0.6(0.9)$ & $0.8(1.1)$ \\
\hline AHEI score & $52.1(10.5)$ & $50.5(10.3)$ & $46.4(10.4)$ & $42.0(10.9)$ \\
\hline \multicolumn{5}{|c|}{$\begin{array}{l}\text { \%E=\% of energy; AHEI=alternate healthy eating index; } \mathrm{BMI}=\text { body mass index; } \mathrm{MET}=\text { metabolic equivalent; } \\
\mathrm{SSB}=\text { sugar sweetened beverage. } \\
{ }^{*} \mathrm{Age} \text { standardized percentages might not be equal to percentages directly calculated by numerator and } \\
\text { denominator in each category of potato consumption. } \\
\text { †Energy adjusted. }\end{array}$} \\
\hline
\end{tabular}

remained significant after additional adjustment for BMI. For French fries, the multivariable adjusted relative risks for GDM for consumption at 1-3 servings/ month, 1 serving/week, and $\geq 2$ servings/week, compared with no consumption or consumption $<1$ serving/month, were 1.07 (0.88 to 1.29 ), 1.25 (1.00 to 1.55 ), and 1.31 (1.00 to 1.70$)$, respectively ( $\mathrm{P}=0.03$ for trend). The association was attenuated and no longer significant after additional adjustment for BMI. We observed a significant and positive correlation between the consumption of different types of potato preparations (that is, baked, boiled, or mashed potatoes, and French fries); the Pearson correlation coefficient was $0.18(\mathrm{P}<0.001)$. This indicates that a woman who ate more baked, boiled, or mashed potatoes was more likely to eat more French fries, and vice versa. To examine the independent associations between different types of potato preparations and GDM, we included both types simultaneously in the same multivariable model and observed almost identical magnitude of the associations with risk. The mutually adjusted relative risks for GDM associated with each increment of one serving/week consumption were 1.08 (1.03 to 1.14) for baked, boiled, or mashed potatoes and 1.08 (1.03 to 1.14) for French fries. For potato chips or corn chips combined, we found no significant association with risk; the multivariable adjusted relative risks for GDM for consumption at 1-3 servings/month, 1 serving/week, and $\geq 2$ servings/week, compared with no consumption or consumption $<1$ serving/month, were 0.97 (0.81 to 1.16), 1.02 (0.83 to 1.25), and 1.00 (0.80 to 1.24), respectively ( $\mathrm{P}=0.87$ for trend).

The association between potato consumption between pregnancy and risk of GDM was robust in multiple sensitivity analyses. First, we observed similar results when we adjusted for individual food items related to diabetes (such as red meat, fruits, vegetables, whole grains, and sugar sweetened beverages), instead of the AHEI diet quality score in the multivariable model (appendix table A). The adjusted relative risks for GDM for total potato consumption of 1, 2-4, and $\geq 5$ servings/week, compared with $<1$ serving/week, were 1.18 (95\% confidence interval 0.95 to 1.45 ), 1.21 (0.99 to 1.48 ), and 1.40 (1.08 to 1.83 ), respectively ( $\mathrm{P}=0.03$ for trend). Second, a sensitivity analysis in which we used the actual continuous amount of total potato consumption, instead of the median level of each category of total potato consumption, in the test of linear trend analysis, yielded almost the same results as those in our main analysis (appendix table B). Third, we observed similar results in a sensitivity analysis that omitted participants with incomplete covariate data, which comprised $4.5 \%$ of the total participants (appendix table $\mathrm{C}$ ). The adjusted relative risks for GDM for total potato consumption of 1, 2-4, and $\geq 5$ servings/week, compared with $<1$ serving/week, were 1.21 (0.97 to 1.50 ), 1.28 (1.04 to 1.57 ), and 1.51 (1.14 to 1.98), respectively ( $\mathrm{P}=0.007$ for trend). Fourth, we also observed similar results in a sensitivity analysis using the most recent pre-pregnancy potato consumption, rather than the cumulative average intake of potatoes, as the exposure variable (appendix table D). The adjusted relative risks for GDM for total potato consumption of 1, 2-4, and $\geq 5$ servings/week, compared with $<1$ serving/week, were 1.17 (0.95 to 1.44), 1.27 (1.04 to 1.55 ), and 1.35 (1.04 to 1.75 ), respectively ( $\mathrm{P}=0.02$ for trend). In addition, we conducted sensitivity analyses by adjusting for major covariates (such as physical activity levels, BMI) as more refined categories (that is, tenths) or as continuous variables (appendix tables E-G). The significant association between total potato consumption and risk of GDM persisted. 


\begin{tabular}{|c|c|c|c|c|c|}
\hline & \multicolumn{4}{|c|}{ Consumption (servings/week) } & \multirow[b]{2}{*}{ P for trend } \\
\hline & $<1$ & 1 & $2-4$ & $\geq 5$ & \\
\hline $\begin{array}{l}\text { GDM/ } \\
\text { pregnancies }\end{array}$ & $136 / 4336$ & $211 / 5763$ & $403 / 9599$ & $195 / 1995$ & - \\
\hline \multicolumn{6}{|l|}{ Models*: } \\
\hline 1 & 1.00 & 1.24 (1.01 to 1.54$)$ & $1.48(1.22$ to 1.79$)$ & 1.93 (1.51 to 2.48$)$ & $<0.001$ \\
\hline 2 & 1.00 & 1.25 (1.01 to 1.54$)$ & 1.45 (1.19 to 1.77$)$ & 1.81 (1.39 to 2.37 ) & $<0.001$ \\
\hline 3 & 1.00 & 1.21 (0.98 to 1.50$)$ & 1.34 (1.10 to 1.64$)$ & 1.62 (1.24 to 2.13$)$ & $<0.001$ \\
\hline 4 & 1.00 & 1.20 (0.97 to 1.48$)$ & 1.27 (1.04 to 1.55$)$ & 1.50 (1.15 to 1.96) & 0.006 \\
\hline \multicolumn{6}{|c|}{$\begin{array}{l}\text { *Model 1: age (months) and parity }(0,1,2, \geq 3 \text { ); model 2: model 1+race/ethnicity (white, African-American, } \\
\text { Hispanic, Asian, others), family history of diabetes (yes, no), cigarette smoking (never, past, current), physical } \\
\text { activity (fourths), and total energy intake (fourths); model 3: model } 2+\text { alternate healthy eating index } 2010 \\
\text { (fourths); model 4: model 3+pre-pregnancy BMI (nine categories; <21, 21-22.9, 23.0-24.9, 25.0-26.9, 27.0-28.9, } \\
\text { 29.0-30.9, 31.0-32.9, 33.0-34.9 and } \geq 35.0 \text { ). }\end{array}$} \\
\hline
\end{tabular}

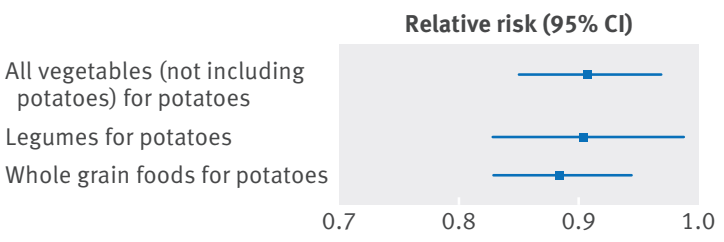

Fig 1 | Risk of gestational diabetes mellitus (GDM) associated with substitution of all vegetables (not including potatoes), legumes, or whole grain foods for potatoes at same serving amount (two servings/week). Adjusted for age (months), parity $(0,1,2, \geq 3)$, race/ ethnicity (white, African-American, Hispanic, Asian, others), family history of diabetes (yes, no), cigarette smoking (never, past, current), physical activity (fourths), total energy intake (fourths), alternate healthy eating index 2010 (fourths), and pre-pregnancy BMI (nine categories: $<21,21-22.9,23.0-24.9,25.0-26.9,27.0-28.9$, $29.0-30.9,31.0-32.9,33.0-34.9$ and $\geq 35.0$ )

\section{Discussion}

In this large prospective cohort study, we found that higher pre-pregnancy consumption of potatoes was significantly associated with a greater risk of gestational diabetes mellitus (GDM), even after adjustment for other major risk factors for GDM such as age, family history of diabetes, physical activity, overall diet quality, and BMI. We estimated that substitution of total potatoes with other vegetables, legumes, or whole grain foods was significantly associated with a lower risk of GDM.

Results in relation to other studies and implications of findings

Although the association between potato consumption and risk of GDM has not been previously investigated, higher potato consumption has been associated with markers of altered glucose metabolism, including increased fasting plasma glucose concentrations and insulin resistance, in people at high risk. ${ }^{8}$ In addition, our results were in line with those from several previous studies, ${ }^{910}$ although not all, ${ }^{3132}$ on the associations of potato consumption with risk of type 2 diabetes mellitus. For instance, a previous study in women who were not pregnant found that higher consumptions of potatoes and French fries was associated with a moderately increased risk of type 2 diabetes mellitus after adjustment for age and dietary and non-dietary factors; women with the highest fifth of consumptions of potatoes and French fries, compared with those with the lowest fifth, had a $14 \%$ and $21 \%$ higher risk, respectively. ${ }^{9}$

Potatoes are widely consumed worldwide. Understanding of the association between consumption and risk of GDM, a common complication of pregnancy that has adverse impacts on the health of women and their children, has important clinical and public health implications. In the US, the Dietary Guidelines for Americans includes potatoes in the vegetable food group, ${ }^{7}$ whereas the United Kingdom's national dietary guidelines classifies potatoes in the starchy food group. ${ }^{33}$ Both guidelines, however, advise people to consume plenty of potatoes as either vegetables or starchy foods. Findings from the present study raise concerns about such guidance. Our findings, together with data from other studies, ${ }^{34-38}$ further highlight the potential role of pre-pregnancy diet in the development of GDM. Future intervention studies are warranted to confirm these findings.

\section{Possible explanations of findings}

Higher consumption of potatoes, in particular French fries, could be an indicator of a low quality diet. We therefore adjusted for quality of overall diet in our analysis. The associations with risk of GDM were moderately attenuated but remained significant after adjustment for overall diet quality. We observed similar results when we adjusted major food groups related to diabetes (that is, red meat, vegetable and fruit, and whole grain foods) and sugar sweetened beverages in place of overall diet quality score (appendix table A). These data suggest that the association between total potato consumption and risk of GDM could be independent of other dietary risk factors for GDM.

Our findings are biologically plausible, though the detailed underlying mechanisms remain to be elucidated. Potatoes are a food with a high glycaemic index because of the large amount of starch that is absorbed rapidly after ingestion. ${ }^{3}$ High potato consumption can result in a sharp postprandial rise in blood glucose concentrations and induce oxidative stress to pancreatic $\beta$ cells and subsequently $\beta$ cell dysfunction or $\beta$ cell exhaustion. ${ }^{39-41}$ Beyond the high glycemic index, French fries also contain altered fatty acid composition and an increased amount of degradation products from the frying oil and dietary advanced glycation end products that are generated during the frying process..$^{4243}$ These changes in fried food have previously been implicated in the development of insulin resistance and diabetes. ${ }^{4445}$ In contrast, other vegetables, legumes, and whole grain foods typically have a low glycemic index, in addition to their richness in vitamins, minerals, fiber, and bioactive phytochemicals; therefore, substitution of potatoes with these foods could lower the risk of GDM. The association between French fries and risk of GDM was substantially attenuated after additional adjustment for BMI, indicating that the association might be largely explained by BMI. This is not surpris- 


\begin{tabular}{|c|c|c|c|c|c|}
\hline & \multicolumn{4}{|c|}{ Consumption (servings/week) } & \multirow{2}{*}{$\begin{array}{l}\mathrm{P} \text { for } \\
\text { trend }\end{array}$} \\
\hline & $<1$ & 1 & $2-4$ & $\geq 5$ & \\
\hline $\begin{array}{l}\text { GDM/ } \\
\text { pregnancies }\end{array}$ & $202 / 5911$ & $274 / 7338$ & $329 / 7493$ & 49/951 & - \\
\hline \multicolumn{6}{|l|}{ Model*: } \\
\hline 1 & 1.00 & 1.18 (0.99 to 1.41$)$ & 1.39 (1.17 to 1.64$)$ & 1.69 (1.24 to 2.28$)$ & $<0.001$ \\
\hline 2 & 1.00 & 1.17 (0.98 to 1.40$)$ & 1.36 (1.13 to 1.63$)$ & 1.63 (1.19 to 2.24$)$ & $<0.001$ \\
\hline 3 & 1.00 & 1.16 (0.97 to 1.39$)$ & 1.30 (1.08 to 1.57$)$ & 1.57 (1.14 to 2.15$)$ & 0.001 \\
\hline 4 & 1.00 & $1.13(0.95$ to 1.35$)$ & $1.25(1.04$ to 1.51$)$ & 1.52 (1.11 to 2.07$)$ & 0.004 \\
\hline \multicolumn{6}{|c|}{ 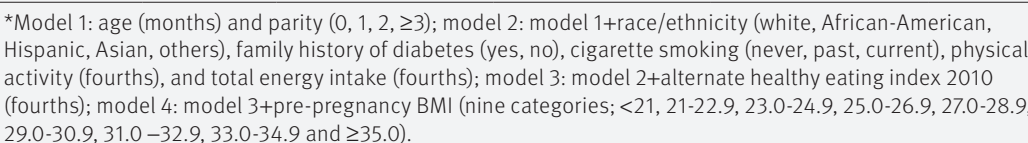 } \\
\hline
\end{tabular}

Table 4 | Relative risks (95\% confidence intervals) for gestational diabetes mellitus according to consumption of French fries before pregnancy

\begin{tabular}{llllll} 
& \multicolumn{3}{l}{ Consumption (servings/week) } & \multirow{2}{*}{ P for trend } \\
\cline { 2 - 5 } & None* & $<1$ & 1 & 21 & - \\
\hline $\begin{array}{l}\text { GDM/ } \\
\text { pregnancies }\end{array}$ & $145 / 4439$ & $383 / 10199$ & $216 / 4884$ & $110 / 2171$ & \\
\hline Modelt: & & & & & \\
\hline 1 & 1.00 & $1.25(1.03$ to 1.51$)$ & $1.62(1.32$ to 2.00$)$ & $1.84(1.45$ to 2.35$)$ & $<0.001$ \\
\hline 2 & 1.00 & $1.18(0.98$ to 1.43$)$ & $1.46(1.18$ to 1.80$)$ & $1.59(1.24$ to 2.04$)$ & $<0.001$ \\
\hline 3 & 1.00 & $1.07(0.88$ to 1.29$)$ & $1.25(1.00$ to 1.55$)$ & $1.31(1.00$ to 1.70$)$ & 0.03 \\
\hline 4 & 1.00 & $1.02(0.84$ to 1.24$)$ & $1.16(0.94$ to 1.45$)$ & $1.18(0.91$ to 1.53$)$ & 0.14 \\
\hline
\end{tabular}

*Includes $<1$ serving/month

tModel 1: age (months) and parity (0, 1, 2, 23); model 2: model 1+race/ethnicity (white, African-American, Hispanic, Asian, others), family history of diabetes (yes, no), cigarette smoking (never, past, current), physical activity (fourths), and total energy intake (fourths); model 3: model 2+alternate healthy eating index 2010 (fourths); model 4: model 3+pre-pregnancy BMI (nine categories; <21, 21-22.9, 23.0-24.9, 25.0-26.9, 27.0-28.9, $29.0-30.9,31.0-32.9,33.0-34.9$ and $\geq 35.0$ ). null. Furthermore, the use of cumulative averages of dietary intakes for participants who filled in more than one pre-pregnancy food frequency questionnaire reduces random error within an individual. ${ }^{26}$ Second, cases of GDM were self reported in this study. Our previous validation study indicated a high degree of accuracy of self reported GDM in this cohort compared with medical record review. ${ }^{17}$ The rate of GDM (5.5\%) in this study was in the upper range of the usual reported rates (range 3-6\%) in the US based on the NDDG (National Diabetes Data Group) criteria during the same period. ${ }^{50} 51$ This rate likely reflected the high rate of screening in this cohort, which is composed of health professionals. Third, we do not have data on the severity of GDM in this cohort. Whether higher habitual consumption of potatoes is associated with increasing severity of GDM warrants future investigation. Fourth, our study population consisted mostly of white American women. Thus, the generalizability of the observed associations might be limited to similar populations. The relative homogeneity of the study population, however, reduces potential confounding due to unmeasured socioeconomic variability. Fifth, although we adjusted for many potential confounders, we are unable to fully rule out the possibility of residual confounding by unmeasured factors. Finally, because of the observational nature of our study, we cannot establish a causal relation between potato consumption and risk of GDM. A carefully conducted observational study, however, could provide a reasonable approach to evaluate the association. In addition, we controlled for major risk factors of GDM to minimize confounding.

ing because frying with oil can increase the energy density of food, ${ }^{46}$ and studies have found an increased risk of obesity associated with higher consumption of fried food. ${ }^{47} 48$ A previous study has shown a significant association between potato consumption and long term weight gain in the general population. ${ }^{49}$ It is possible that eating more potatoes is related to higher gestational weight gain and, subsequently, an increased risk of GDM. Unfortunately, we did not have data on gestational weight gain to further explore the potential mechanism.

\section{Strengths and potential limitations}

The strengths of this study include the prospective design with a long follow-up that establishes the temporal direction of the associations, the large number of cases of GDM that ensures sufficient statistical power, and the repeated and comprehensive assessment of many dietary and lifestyle variables that allows us to assess potential confounding and effect modification. We acknowledge that there were also several potential limitations. First, like other observational studies, measurement error in self reported exposures (potato consumption) is possible. As the pre-pregnancy dietary information was captured prospectively, the random error within an individual would most likely be non-differential, which could have attenuated the observed associations toward

\section{Conclusion}

In summary, in this large prospective cohort study, pre-pregnancy potato consumption was significantly and positively associated with the risk of incident GDM. Substitution of potatoes with other vegetables, legumes, or whole grain foods was associated with a lower risk of GDM. These findings should be confirmed in intervention studies and randomized controlled trials. were involved in data collection. WB analyzed the data and drafted the to the interpretation of the results and revision of the manuscript for important intellectual content and approved the final version of the manuscript. WB and $\mathrm{CZ}$ are guarantors.

Funding: This study was supported by the Intramural Research Program of the Eunice Kennedy Shriver National Institute of Child Health and Human Development, National Institutes of Health (contract No HHSN275201000020C). The Nurses' Health Study II was funded by research grants DK58845, CA50385, P30 DK46200, and UM1 CA176726 from the National Institutes of Health. DKT and FBH were supported by a mentored fellowship from the American Diabetes Association (No 7-12-MN-34)

Competing interest: All authors have completed the ICMJE uniform disclosure form at www.icmje.org/coi_disclosure.pdf and declare: no support from any organization for the submitted work; no financial relationships with any organizations that might have an interest in the submitted work in the previous three years; no other relationships or activities that could appear to have influenced the submitted work.

Ethical approval: The study protocol was approved by the Partners Human Research Committee (Boston, MA, USA). The completion of the self administered questionnaire was considered to imply informed consent.
Contributors: WB and CZ contributed to the study design. JEC and FBH manuscript. DKT conducted technical review. All authors contributed 
Transparency: The senior author, CZ, affirms that the manuscript is an honest, accurate, and transparent account of the study being reported; that no important aspects of the study have been omitted.

Data sharing: No additional data available.

This is an Open Access article distributed in accordance with the Creative Commons Attribution Non Commercial (CC BY-NC 3.0) license, which permits others to distribute, remix, adapt, build upon this work non-commercially, and license their derivative works on different terms, provided the original work is properly cited and the use is noncommercial. See: http://creativecommons.org/licenses/by-nc/3.0/.

1 Camire ME, Kubow S, Donnelly DJ. Potatoes and human health. Crit Rev Food Sci Nutr 2009;49: 823-40

doi:10.1080/10408390903041996 19960391

2 Freedman MR, Keast DR. Potatoes, including French fries, contribute key nutrients to diets of U.S. adults: NHANES 2003-2006. / Nutr Ther 2012;1:1-11.

3 McGill CR, Kurilich AC, Davignon I. The role of potatoes and potato components in cardiometabolic health: a review. Ann

Med 2013;45: 467-73. doi:10.3109/07853890.2013.813633 23855880

4 Willett WC. The dietary pyramid: does the foundation need repair? Am J Clin Nutr 1998:68: 218-9, 9701173

5 Forbes GB. The potato's placement in the dietary pyramid. Am I Clin Nutr 1999;69: 572-3.10075348

6 King IC, Slavin JL. White potatoes, human health, and dietary guidance. Adv Nutr 2013;4: 393S-401S. doi:10.3945/ an.112.003525 23674809

7 Center for Nutrition Policy and Promotion. Dietary Guidelines for Americans, 2010. 7th edU.S. Department of Agriculture and U.S. Department of Health and Human Services, 2010. http://www.cnpp. usda.gov/DietaryGuidelines.

8 Ylönen SK, Virtanen SM, Groop LBotnia Research Group. The intake of potatoes and glucose metabolism in subjects at high risk for Type 2 diabetes. Diabet Med 2007;24: 1049-50.

doi:10.1111/i.1464-5491.2007.02206.x 17725708

9 Halton TL, Willett WC, Liu S, Manson JE, Stampfer MJ, Hu FB. Potato and french fry consumption and risk of type 2 diabetes in women. Am J Clin Nutr 2006;83: 284-90. 16469985

10 Montonen I, Järvinen R, Heliövaara M, Reunanen A, Aromaà A, Knekt P. Food consumption and the incidence of type II diabetes mellitus. Eur/ Clin Nutr 2005;59: 441-8. doi:10.1038/ si.ejcn.160209415674312

11 American Diabetes Association. Gestational diabetes mellitus. Diabetes Care 2004;27(Suppl 1): S88-90. doi:10.2337/ diacare.27.2007.S88 14693936

12 Metzger BE, Lowe LP, Dyer ARHAPO Study Cooperative Research Group. Hyperglycemia and adverse pregnancy outcomes. N EnglJ Med 2008;358:1991-2002. doi:10.1056/NEJMoa0707943 18463375

13 Reece EA, Leguizamón G, Wiznitzer A. Gestational diabetes: the need for a common ground. Lancet 2009;373: 1789-97. doi:10.1016/ S0140-6736(09)60515-8 19465234

14 Scholl TO, Chen X, Khoo CS, Lenders C. The dietary glycemic index during pregnancy: influence on infant birth weight, fetal growth, and biomarkers of carbohydrate metabolism. Am J Epidemiol 2004;159: 467-74. doi:10.1093/aje/kwh068 14977642

15 Tieu J, Crowther CA, Middleton P. Dietary advice in pregnancy for preventing gestational diabetes mellitus. Cochrane Database Syst Rev 2008;(2): CD006674. 18425961

16 Zhang C, Liu S, Solomon CG, Hu FB. Dietary fiber intake, dietary glycemic load, and the risk for gestational diabetes mellitus. Diabetes Care 2006;29: 2223-30. doi:10.2337/dc06-0266 17003297

17 Solomon CG, Willett WC, Carey VJ. A prospective study of pregravid determinants of gestational diabetes mellitus. JAMA 1997;278: 1078-83. doi:10.1001/jama.1997.035501300520369315766

18 Colditz GA, Hankinson SE. The Nurses' Health Study: lifestyle and health among women. Nat Rev Cancer 2005;5: 388-96. doi:10.1038/ $\operatorname{nrc1608} 15864280$

19 Willett WC, Sampson L, Stampfer MJ. Reproducibility and validity of a semiquantitative food frequency questionnaire. $\mathrm{Am}$ Epidemiol 1985:122: 51-65. 4014201

20 Willett WC, Sampson L, Browne ML. The use of a self-administered questionnaire to assess diet four years in the past. Am J Epidemiol 1988;127: 188-99. 3337073

21 Salvini S, Hunter DJ, Sampson L. Food-based validation of a dietary questionnaire: the effects of week-to-week variation in food consumption. IntJ Epidemiol 1989;18: 858-67. doi:10.1093/ iie/18.48582621022

22 National Diabetes Data Group. Classification and diagnosis of diabetes mellitus and other categories of glucose intolerance. Diabetes 1979;28: 1039-57. doi:10.2337/diab.28.12.1039510803

23 Rimm EB, Stampfer MJ, Colditz GA, Chute CG, Litin LB, Willett WC. Validity of self-reported waist and hip circumferences in men and women. Epidemiology 1990;1: 466-73. doi:10.1097/00001648-199011000-00009 2090285
24 Wolf AM, Hunter DJ, Colditz GA. Reproducibility and validity of a self-administered physical activity questionnaire. Int J Epidemiol 1994:23: 991-9. doi:10.1093/ije/23.5.9917860180

25 Chiuve SE, Fung TT, Rimm EB. Alternative dietary indices both strongly predict risk of chronic disease. J Nutr 2012;142: 1009-18. doi:10.3945/in.111.157222222513989

26 Hu FB, Stampfer MJ, Rimm E. Dietary fat and coronary heart disease: a comparison of approaches for adjusting for total energy intake and modeling repeated dietary measurements. Am J Epidemiol 1999;149: 531-40. doi:10.1093/oxfordjournals.aje. a00984910084242

27 Wacholder S. Binomial regression in GLIM: estimating risk ratios and risk differences. Am J Epidemiol 1986:123: 174-84. 3509965

28 Hanley JA, Negassa A, Edwardes MD, Forrester JE. Statistical analysis of correlated data using generalized estimating equations: an orientation. Am J Epidemiol 2003;157:364-75. doi:10.1093/aje/ kwf215 12578807

29 Zou G. A modified poisson regression approach to prospective studies with binary data. Am J Epidemiol 2004;159: 702-6. doi:10.1093/aje/kwh090 15033648

30 Pan A, Sun Q, Bernstein AM. Red meat consumption and mortality: results from 2 prospective cohort studies. Arch Intern Med 2012:172: 555-63. doi:10.1001/ archinternmed.2011.2287 22412075

31 Liu S, Serdula M, Janket SJ. A prospective study of fruit and vegetable intake and the risk of type 2 diabetes in women. Diabetes Care 2004:27: 2993-6. doi:10.2337/diacare.27.12.2993 15562224

32 Hodge AM, English DR, O'Dea K, Giles GG. Glycemic index and dietary fiber and the risk of type 2 diabetes. Diabetes Care 2004;27: 2701-6. doi:10.2337/diacare.27.11.2701 15505008

33 UK National Health Service. The eatwell plate. http://www.nhs.uk/ Livewell/Goodfood/Pages/eatwell-plate.aspx.

34 Zhang C, Ning Y. Effect of dietary and lifestyle factors on the risk of gestational diabetes: review of epidemiologic evidence. Am J Clin Nutr 2011;94(Suppl): 1975S-9S. doi:10.3945/ ajcn.110.001032 21613563

35 Tobias DK, Zhang C, Chavarro J. Prepregnancy adherence to dietary patterns and lower risk of gestational diabetes mellitus. Am J Clin Nutr 2012;96: 289-95. doi:10.3945/ajcn.111.028266 22760563

36 Bao W, Bowers K, Tobias DK, Hu FB, Zhang C. Prepregnancy dietary protein intake, major dietary protein sources, and the risk of gestational diabetes mellitus: a prospective cohort study. Diabetes Care 2013;36: 2001-8. doi:10.2337/dc12-2018 23378620

37 Bao W, Bowers K, Tobias DK. Prepregnancy low-carbohydrate dietary pattern and risk of gestational diabetes mellitus: a prospective cohort study. Am J Clin Nutr 2014;99: 1378-84. doi:10.3945/ ajcn.113.082966 24717341

38 Schoenaker DA, Soedamah-Muthu SS, Callaway LK, Mishra GD. Prepregnancy dietary patterns and risk of gestational diabetes mellitus: results from an Australian population-based prospective cohort study. Diabetologia 2015:58: 2726-35. doi:10.1007| s00125-015-3742-1 26358582

39 Riccardi G, Rivellese AA, Giacco R. Role of glycemic index and glycemic load in the healthy state, in prediabetes, and in diabetes. Am J Clin Nutr 2008;87: 269S-74S. 18175767

40 Willett W, Manson J, Liu S. Glycemic index, glycemic load, and risk of type 2 diabetes. Am I Clin Nutr 2002;76: 274S-80S. 12081851

41 Ceriello A, Esposito K, Piconi L. Oscillating glucose is more deleterious to endothelial function and oxidative stress than mean glucose in normal and type 2 diabetic patients. Diabetes 2008;57:1349-54. doi:10.2337/db08-006318299315

42 Guallar-Castillón P, Rodríguez-Artalejo F, Lopez-

Garcia E. Consumption of fried foods and risk of coronary heart disease: Spanish cohort of the European Prospective Investigation into Cancer and Nutrition study. BMJ 2012;344: e363. doi:10.1136/ bmj.e36322275385

43 Goldberg T, Cai W, Peppa M. Advanced glycoxidation end products in commonly consumed foods. I Am Diet Assoc 2004:104: 1287-91. doi:10.1016/j.jada.2004.05.21415281050

44 Cai W, Ramdas M, Zhu L, Chen X, Striker GE, Vlassara H. Oral advanced glycation endproducts (AGEs) promote insulin resistance and diabetes by depleting the antioxidant defenses AGE receptor-1 and sirtuin 1. Proc Natl Acad Sci U S A 2012;109: 15888-93. doi:10.1073/ pnas.1205847109 22908267

45 Cahill LE, Pan A, Chiuve SE. Fried-food consumption and risk of type 2 diabetes and coronary artery disease: a prospective study in 2 cohorts of US women and men. Am I Clin Nutr 2014;100: 667-75. doi:10.3945/ajcn.114.084129 24944061

46 Pokorn J, Pánek J, Trojáková L. Effect of food component changes during frying on the nutrition value of fried food. Forum Nutr 2003;56: 348-50. 15806930

47 Guallar-Castillón P, Rodríguez-Artalejo F, Fornés NS. Intake of fried foods is associated with obesity in the cohort of Spanish adults from the European Prospective Investigation into Cancer and Nutrition. Am J Clin Nutr 2007;86: 198-205. 17616781 
48 Qi Q, Chu AY, Kang JH. Fried food consumption, genetic risk, and body mass index: gene-diet interaction analysis in three US cohort studies. BM/2014:348: g1610. 24646652

49 Mozaffarian D, Hao T, Rimm EB, Willett WC, Hu FB. Changes in diet and lifestyle and long-term weight gain in women and men. N Engl J Med 2011:364: 2392-404. doi:10.1056/ NEJMoa1014296 21696306

50 Magee MS, Walden CE, Benedetti TJ, Knopp RH. Influence of diagnostic criteria on the incidence of gestational diabetes and perinatal morbidity. JAMA 1993;269: 609-15. doi:10.1001/ jama.1993.035000500870318421365

51 Ferrara A, Hedderson MM, Quesenberry CP, Selby JV. Prevalence of gestational diabetes mellitus detected by the national diabetes data group or the carpenter and coustan plasma glucose thresholds. Diabetes Care 2002;25: 1625-30. doi:10.2337/diacare.25.9.162512196438 (C) BMJ Publishing Group Ltd 2016

Appendix: Supplementary tables A-G 